\title{
Somatic symptom disorder treated with electroconvulsive therapy
}

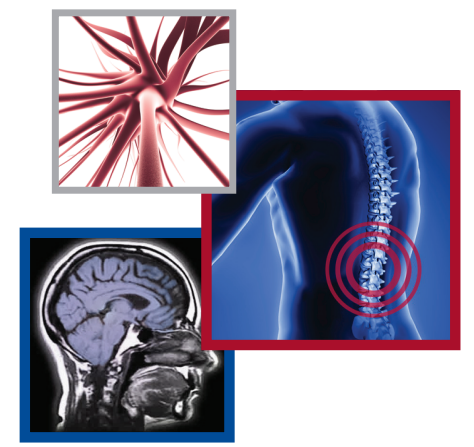

\author{
Anna Borisovskaya* ${ }^{* 1,2}$ \& Jay Alan Augsburger ${ }^{1}$
}

\section{Practice points}

- Preliminary evidence suggests that electroconvulsive therapy should be considered for treatment of somatic symptom disorder comorbid with major depressive disorder, when standard treatments fail.

- Larger, well-designed research studies are necessary to further evaluate the impact of electroconvulsive therapy in the treatment of somatic symptom disorder and chronic pain conditions.

Somatic symptom disorder (SSD) is a challenging condition to treat with chronic pain, a common and disabling symptom. We present a patient who received electroconvulsive therapy (ECT) for SSD with significant improvement in pain and gastrointestinal symptoms. We also present a brief literature review of similar cases treated with ECT. Preliminary evidence suggests that ECT should be considered for treatment of SSD comorbid with major depressive disorder, when standard treatments fail. Further research is needed to clarify whether ECT can be used for SSD without associated depression.

First draft submitted: 30 August 2016; Accepted for publication: 2 November 2016; Published online: 23 November 2016

Somatic symptom disorder (SSD), as defined by the Diagnostic and Statistical Manual of Mental Disorders, Fifth Edition, is characterized by the somatic symptoms that are distressing or significantly disruptive in daily life. This condition presents with excessive thoughts, feelings or behaviors related to the somatic symptoms, with attendant high levels of anxiety about one's health, excessive time and energy devoted to one's symptoms, and/or disproportionate and persistent thoughts about the seriousness of one's symptoms, persisting for 6 months or more. Often, SSD presents with chronic pain. We discuss Ms B, a 55-year-old woman with SSD and comorbid major depressive disorder (MDD) who was treated successfully with electroconvulsive therapy (ECT).

Ms B was admitted to the inpatient psychiatry unit at the Veterans Affairs hospital with worsening nausea and abdominal pain, accompanied by weight loss of 40 pounds in the preceding 5 months. Her gastrointestinal symptoms were so severe that she was unable to eat or work. Her medical history was significant for a cholecystectomy performed 11 years ago. A total of 1 year prior to the hospitalization, she was successfully treated for a gastric ulcer related to Helicobacter pylori infection. Medical workup of her current gastrointestinal symptoms was thorough; it included a number of imaging studies, including abdominal plain films, esophagogastroduodenoscopy, colonoscopy and magnetic resonance

'Mental Health Service, Veterans Affairs Medical Center, Seattle, WA, USA

${ }^{2}$ Department of Psychiatry \& Behavioral Sciences, University of Washington, Seattle, WA, USA

*Author for correspondence: Tel.: +1 206277 1926; Fax: +1 206764 2946; anna.borisovskaya@va.gov

\section{KEYWORDS}

- chronic pain

- electroconvulsive therapy

- somatic symptom disorder 
enterography. No medical explanation was found for her symptoms. Pantoprazole and anti-emetics were ineffective in relieving her distress.

Prior to the onset of gastrointestinal symptoms, her psychiatric history had been unremarkable. However, after being diagnosed with a gastric ulcer, she developed depressed mood and anxiety. Subsequently, she attempted suicide by overdose but fortunately survived. She was started on fluoxetine $40 \mathrm{mg}$ daily, though reported no improvement in her mood, anxiety or somatic symptoms despite taking the medication for 1 year. Of note, fluoxetine was initiated after the onset of her gastrointestinal distress, and therefore did not appear to be the cause of her nausea. Shortly prior to the admission to the Veterans Affairs hospital, she was treated for 1 week at an outside psychiatric hospital for suicidal ideation, with no improvement in her mood.

Upon her admission to the Veterans Affairs hospital, the patient's Beck Depression Inventory, 2nd edition was scored at 56 , indicating severe depression. Her mental status exam was notable for poor eye contact, psychomotor agitation, catastrophic thinking process and apparent physical discomfort. She complained of severe anxiety. She was too distressed to participate in psychotherapy or in the unit programming. She gained minimal relief from as needed doses of lorazepam. During the hospitalization, fluoxetine was increased to $60 \mathrm{mg}$, and ondansetron was added for nausea, with no improvement.

ECT was recommended because of poor response to medications, severity of her depression and persistent suicidal ideation. She consented to treatment. ECT was administered three-times a week with brief pulse constant current stimulation using a Thymatron System IV (Somatics, LLC, Lake Bluff, IL, USA). The stimulus setting of $100 \%$ of the maximal charge was implemented $(100 \%$ for the Thymatron machine $=504 \mathrm{mC})$. Right unilateral electrode placement with ultrabrief pulse $(0.3 \mathrm{~ms})$ was used. Anesthesia was implemented with methohexital $90 \mathrm{mg}$ intravenously and succinylcholine $80 \mathrm{mg}$ intravenously. An electroencephalogram was recorded during the treatment. Cardiac rhythm, blood pressure, heart rate and oxygenation were monitored before, during and after the treatments. Her seizures averaged 30-60 s in duration with high amplitude spike and wave activity and excellent postictal suppression. After the first two treatments, she experienced significant bradycardia which was effectively treated with $0.2 \mathrm{mg}$ intravenous dose of glycopyrrolate, but this complication did not recur during subsequent procedures.

Her mood began to improve after the third session. After the fifth session of ECT, her mood had improved her nausea and pain dissipated, and she did not request anti-emetics for the rest of her hospitalization. She continued ECT for a total of seven sessions, achieving remission of her depression (Beck Depression Inventory, 2nd edition score of 7) and significant relief in her gastrointestinal pain by the time of discharge. Her suicidal ideation resolved. Based on the severity of her presentation, she was offered maintenance ECT to prevent recurrences of her depression and pain, but she declined. Two months after the completion of the index course, she had not experienced recurrence of her gastrointestinal symptoms and her depression remained in remission.

\section{Discussion}

A description of ECT as being helpful in somatic symptoms and pain disorders dates back to 1968, when 66 inpatients with hypochondriasis were treated with ECT, with reported improvement rate of $50 \%$ [1]. Several case reports and studies supporting the use of ECT to treat SSD and related diagnoses have been published since. For example, a patient who suffered from the somatization disorder for 30 years, experienced significant improvement in his symptoms after five sessions of ECT, with negligible effect on his anxiety and depression [2]. A 72-year-old woman with a hypochondriacal delusion experienced complete remission during an index course of ECT; again, she did not have concomitant affective illness. She required maintenance ECT for recurrence of her symptoms 2.5 years after the index course [3]. A woman in her late forties with MDD and idiopathic burning mouth syndrome (in Diagnostic and Statistical Manual of Mental Disorders, Fifth Edition [DSM-5], this would be classified as SSD) experienced resolution of her symptoms after ten-session index course of ECT, and had continuation ECT treatment for another ten sessions, with sustained benefit [4]. Another burning mouth syndrome patient without MDD experienced substantial improvement in her pain after 12 bilateral ECT sessions, with no worsening at 2 years' time post treatment [5]. A 71-year-old woman with globus hystericus had such severe depressive symptoms that the authors hypothesized that swallowing difficulty was part of the depressive illness in itself. Her complaint of globus resolved completely after three sessions of ECT [6]. 
The largest study to date was a retrospective case series of 28 patients who received ECT for SSD. Of these 28 patients, 21 had pseudoneurologic symptoms, 14 had pain symptoms and two had significant gastrointestinal symptoms as well as extremity weakness. All of the patients in these subgroups had comorbid MDD. Encouraging results were seen in all groups, where $86 \%$ of patients with pseudoneurologic symptoms, 79\% of patients with pain and $50 \%$ of patients with gastrointestinal symptoms reported improvement. Notably, $21 \%$ of patients of those who had pain symptoms reported worsening of their pain following ECT [7]. Another study with a relatively larger sample size documents improvement of intractable pain and refractory depression in $95 \%$ of patients during the course of ECT [8].

Chronic regional pain syndrome (CRPS) has also been successfully addressed by ECT. A 49-year-old patient with failed back syndrome and CRPS, as well as MDD, responded to ECT where she had not responded to standard treatments. It did take three acute ECT courses and the pain improved only at the end of the third course. Her depression improved after the first course, though a 1 month period of time without ECT led to a relapse [9]. In another study, three patients with CRPS and concomitant depression were described as responsive to ECT, both for improvement of depression and for resolution of pain. Interestingly, the patient who was also diagnosed with fibromyalgia did not improve [10]. Another neuropathic condition, brachial plexopathy in a 32-year-old male with comorbid MDD was also successfully managed with ECT, the effect of which lasted for 8 weeks [11].

The most recent report documents a woman in the seventh decade of her life with chronic right upper quadrant pain, which was diagnosed as neuropathic and unresponsive to all treatments tried. She also likely had MDD. Three acute courses of ECT led to analgesia each time [12].

The mechanism by which ECT improves chronic pain is not clear. ECT is known to affect cerebral metabolism, in the frontal, parietal, left temporal cortexes and in the cingulate gyrus, one of the putative mechanisms of its antidepressant effect [13]. Changes in the regional cerebral blood flow to thalamus have also been demonstrated in a chronic pain patient whose symptoms markedly improved after ECT [14]. ECT also causes changes in the availability of neurotransmitters and causes a consistent increase in the amount of endorphins and dynorphines in the central nervous system; further, it leads to cell growth and increase in synaptic connectivity $[15,16]$. ECT also appears to increase pain threshold and pain tolerance in patients with MDD, along with improvements in depressive symptoms [17].

\section{Conclusion}

Literature reviewed above suggests that ECT can be effective in treating patients with SSD and pain disorders. Excellent response to ECT has been described in neuropathic pain conditions, such as burning mouth syndrome, brachial plexopathy and CRPS, as well as in treating unexplained gastrointestinal pain. Unilateral and bilateral methods of ECT administration were described as being effective in the literature reviewed above; courses varied significantly in length. Some failures have also been described, such as in the treatment of fibromyalgia. Given the publication bias, it is likely that a number of other negative experiences have not made it into the literature. While in the case we described, improvement in depression preceded improvement in abdominal pain, it is important to note that patients without comorbid affective symptoms have also experienced relief of their somatic symptoms, in the cases described above.

We conclude, based on the preliminary evidence reviewed above, that ECT should be considered as a treatment option for patients with SSD, particularly in the presence of comorbid depression and suicidal ideation, and particularly if their somatic symptoms include pain. It should be remembered that improvements in depression and pain may not occur simultaneously; one may improve before the other. At this time, it is too early to recommend that SSD and pain disorders without concomitant affective illness be treated with ECT. Randomized controlled trials of ECT for SSD and pain disorders should be completed; ECT compared with sham ECT for these conditions without comorbid affective disorders would be particularly useful.

Financial \& competing interests disclosure

The authors have no relevant affliations or financial involvement with any organization or entity with a financial interest in or financial conflict with the subject matter or materials discussed in the manuscript. This includes employment, consultancies, honoraria, stock ownership or options, expert testimony, grants or patents received or pending, or royalties.

No writing assistance was utilized in the production of this manuscript. 


\section{References}

Papers of special note have been highlighted as: - of interest; $\bullet \bullet$ of considerable interest

1 Pilowsky I. The response to treatment in hypochondriacal disorders. Aust. NZ J. Psychiatry 2(2), 88-94 (1968).

2 Gahr M, Schoenfeldt-Lecuona C, Connemann BJ. Somatization disorder treated with electroconvulsive therapy. J. ECT 27(3), 266-267 (2011).

3 Dols A, Rhebergen D, Eikelenboom P, Stek ML. Hypochondriacal delusion in an elderly woman recovers quickly with electroconvulsive therapy. Clin. Pract. 2(1), e11 (2012)

4 McGirr A, Davis L, Vila-Rodriguez F. Idiopathic burning mouth syndrome: a common treatment-refractory somatoform condition responsive to ECT. Psychiatry Res. 216, 158-159 (2014).

5 Suda S, Takagai S, Inoshima-Takahashi K, Sugihara G, Mori N, Takei N.

Electroconvulsive therapy for burning mouth syndrome. Acta Psychiatr. Scand. 118, 503-504 (2008)

6 Cybulska EM. Globus hystericus - a somatic symptom of depression? The role of electroconvulsive therapy and antidepressants. Psychosom. Med. 59, 67-69 (1997).

7 Leong K, Tham JC, Scamvougeras A, Vila-Rodriguez F. Electroconvulsive therapy treatment in patients with somatic symptom and related disorders. Neuropsychiatr. Dis. Treat. 11, 2565-2572 (2015).

- The largest study to date of treating somatic symptom disorder with associated depression.

8 Bloomstein JR, Rummans TA, Maruta T,Lin SC, Pileggi TS., The use of electroconvulsive therapy in pain patients. Psychosomatics 37, 374-379 (1996).

9 Suzuki K, Ebina Y, Shindo T, Takano T, Awata S, Matsuoka H.Repeated electroconvulsive therapy courses improved chronic regional pain with depression caused by failed back syndrome. Med. Sci. Monit. 15(4), CS77-CS79 (2009).

10 McDaniel WW. Electroconvulsive therapy in complex regional pain syndromes. J. ECT 19, 226-229 (2003).

11 Abdi S, Haruo A, Bloomstone J. Electroconvulsive therapy for neuropathic pain: a case report and literature review. Pain Physician 7, 261-263 (2004).

- Offers a literature review of electroconvulsive therapy (ECT) in neuropathic pain conditions.

12 Cooper JJ. Recurrent right upper quadrant pain responsive only to electroconvulsive therapy. J. ECT 32(3), e21-e22 (2016).

13 Nobler MS, Oquendo MA, Kegeles LS et al. Decreased regional brain metabolism after
ECT. Am. J. Psychiatry 158(2), 305-308 (2001).

14 Fukui S, Shigemori S, Yoshimura A, Nosaka S. Chronic pain with beneficial response to electroconvulsive therapy and regional cerebral blood flow changes assessed by single photon emission computed tomography. Reg. Anesth. Pain Med. 27(2), 211-213 (2002).

-• A nicely done functional imaging study demonstrating changes in cerebral blood flow after ECT.

15 Wahlund B, von Rosen D. ECT of major depressed patients in relation to biological and clinical variables: a brief overview. Neuropsychopharmacology 28, S21-S26 (2003).

- An overview of ECT effects in major depressive disorder.

16 Holaday JW, Tortella FC, Meyerhoff JL,Belenky GL,Hitzemann RJ.

Electroconvulsive shock activates endogenous opioid systems: behavioral and biochemical correlates. Ann. NY Acad. Sci. 467, 249-255 (1986).

17 Schreiber S, Shmueli D, Grunhaus L et al. The influence of electroconvulsive therapy on pain threshold and pain tolerance in major depression patients before, during and after treatment. Eur. J. Pain 7, 419-424 (2003). 